\title{
A psychoanatomical investigation of the locus of the mechanism responsible for the refractoriness of the visual vertex potential
}

\author{
D. G. WASTELL \\ MRC Applied Psychology Unit, I5 Chaucer Road, Cambridge, England \\ and \\ D. KLEINMAN \\ University of Durham, Durham, England
}

\begin{abstract}
The anatomical locus of the refractoriness of the N1-P2 wave of the visual vertex evoked potential is examined using the dichoptic viewing paradigm (Turvey, 1973). N1-P2 amplitude to the second stimulus of a pair delivered to the eye contralateral to the first stimulus is found to exhibit the same amplitude depression as a second stimulus delivered to the ipsilateral eye. A central locus for N1-P2 refractoriness is inferred from this result.
\end{abstract}

The vertex potential (N1-P2) of the human scalprecorded evoked potential (EP) exhibits a state of refractoriness which persists for an interval of several seconds following its evocation. Depending upon the particular paradigm being used, this refractoriness is described as either the "recovery process" (Davis, Mast, Yoshie, \& Zerlin, 1966) or "short-term habituation" (Fruhstorfer, Soveri, \& Jarvilehto, 1970). Although most of the research has been conducted within the auditory modality, the vertex potential to visual and somatosensory stimuli also demonstrates refractoriness (Davis, Osterhammel, Weir, \& Gjerdingen, 1972), although the phenomenon is less marked in the visual system (Gjerdingen \& Tomsic, 1970).

In the auditory modality, Bess and Ruhm (1972), using dichotic presentation, located the neural nets responsible for the refractoriness of the auditory vertex potential at a level beyond binaural convergence. The present work similarly employs the dichoptic viewing paradigm (Turvey, 1973) to examine the locus of the refractory nets responsible for the phenomenon of the refractoriness of the visual vertex potential. In man, although some interactions between inputs from the two eyes do exist at the level of the lateral geniculate body (Szengothai \& Arbib, 1975), binocular convergence is generally associated with a cortical level of processing, and the dichoptic paradigm has been widely employed to investigate whether the mechanisms of visual phenomena are peripheral (precortical) or central (cortical). Wastell and Kleinman (Note 1) have shown, using an arti-

This research was conducted at the Department of Psychology, University of Durham. The financial support of the SRC is acknowledged. ficial pupil, that a pupillary mechanism does not subserve the refractoriness of the visual EP, and the present application of the dichoptic viewing paradigm continues this psychoanatomical investigation.

\section{METHOD}

\section{Subjects and Recordings}

Six subjects, all postgraduates at Durham University, participated in the experiment. EEG was recorded monopolarly (referred to an earlobe placement) with $\mathrm{Ag}-\mathrm{AgCl}$ electrodes from a vertex electrode placement $\left(C_{z}, 10 / 20\right.$ electrode system). Amplification was performed with a Grass 7 P58 preamplifier (time constant $=.1 \mathrm{sec}$, upper cutoff $=500 \mathrm{~Hz}$ ) coupled to a 7DAF driver module. Digitization of the EEG proceeded on-line at a rate of $1 \mathrm{pt} / 5 \mathrm{msec}$, and epochs $500 \mathrm{msec}$ in duration were collected for off-line analysis on an IBM 370 computer.

\footnotetext{
Apparatus

Using the apparatus depicted in Figure 1, either eye could be monocularly stimulated with a light-emitting diode (LED) flash (10 msec duration). Two red LEDs were mounted on opposite sides of a midline at the focal length of the two lenses, $A_{1}$ and $A_{2}$. Parallel light from both sources was arranged to be directed onto a third lens, B, whose focal place coincided with the subject's frontal plane. With this optical arrangement, flashing of the LED $A_{1}$ thus produced monocular stimulation of the right eye and, conversely, $A_{2}$ monocularly stimulated the left eye. Monocularity was confirmed by covering the right eye for an $A_{1}$ flash and the left eye for $A_{2}$. Under these conditions, flashes were not observable. At this point, it should be noted that the red light, D, was used to provide diffuse low-intensity background lighting which was effective in obscuring any unwanted stray light, including that scattering off the front surface of $B$.

From the subjects' point of view, stimulation consisted of the brief uniform illumination of the aperture $\mathrm{C}$ (visual angle = $.75 \mathrm{deg}$ ). Prior to each experimental session, the perceptual equality of right- and left-eye stimulation was checked. Following minor adjustments to the apparatus for two subjects, all subjects reported no difference between right and left stimulation. LED and background luminances were measured at the eye, using a SEI spot photometer, with values of 8.5 and $.1 \mathrm{~cd} / \mathrm{m}^{2}$ being obtained, respectively.
} 


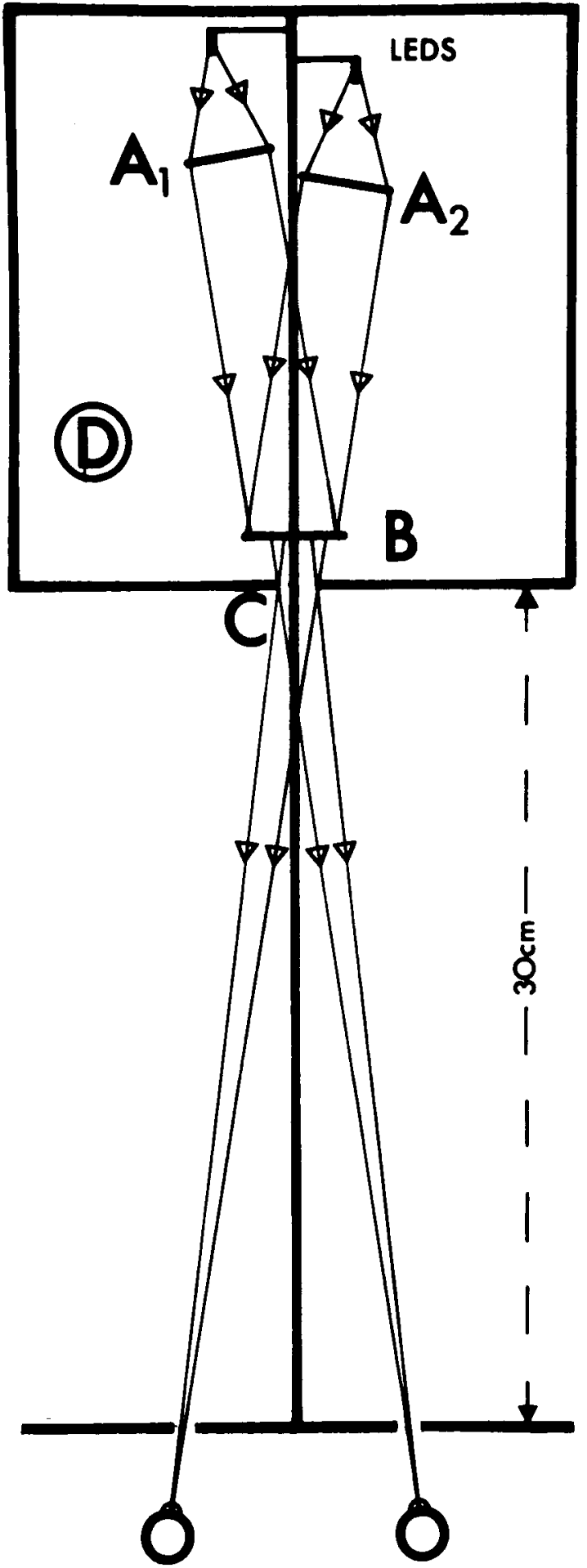

Figure 1. The apparatus of the experiment viewed from above. See text for description.

\section{Procedure}

One hundred pairs of stimuli (interpair interval, IPI = $10 \mathrm{sec}$; interstimulus interval, ISI $=1 \mathrm{sec}$ ), presented in two blocks of 50, constituted the experimental session. Previous work (Wastell, $1978 \mathrm{a}$, pp. 168-172) had indicated that visual refractoriness is effectively dissipated after a "silent" interval of $10 \mathrm{sec}$. This is in accord with the literature (Gjerdingen \& Tomsic, 1970, for instance, find the visual recovery function to effectively flatten by the 10-sec point) and confirms the suitability of the 10-sec IPI.

Four types of stimulus pairs-corresponding to the combinations: left eye first stimulus, right eye second; left first, left second; right first, left second; and right first, right second-were used, the 100 pairs of the session representing a random permutation of 25 of each type. The subjects were required to fixate the aperture $\mathrm{C}$ throughout and were instructed to attend passively to the flashes.

\section{RESULTS}

Three average EPs were computed for each subject representing the brain activity evoked by the first stimulus of a pair (1), the second stimulus of a pair delivered to the eye ipsilateral to the first stimulus (2i), and the second stimulus of a pair delivered to the eye contralateral to the first $(2 \mathrm{c})$. These averages were smoothed using a digital filter $(-3 \mathrm{~dB}$ at $20 \mathrm{~Hz}$ ) designed to eliminate without phase distortion unwanted high-frequency noise from waveforms (Wastell, 1978b). The filtered waveforms for all subjects are presented for illustrative purposes in Figure 2. N1 and P2 were objectively identified in all records as the most negative-going and positive-going

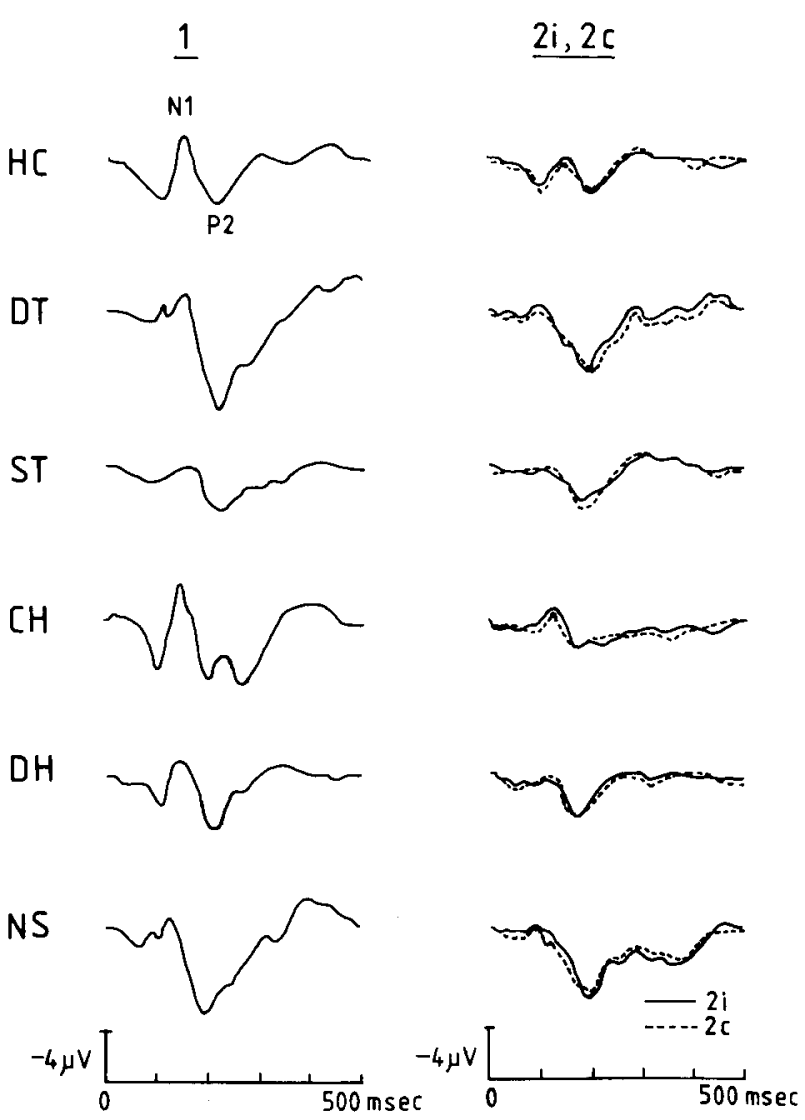

Figure 2. Digitally filtered average EPs for all subjects associated with the first stimulus of a pair (1), an ipsilateral second stimulus ( $2 \mathrm{i})$, and a contralateral second stimulus (2c). Note that waveforms for $2 i$ and $2 c$ are shown superimposed in the righthand column, with $2 \mathrm{i}$ represented by the continuous line and $2 \mathrm{c}$ by the broken line. 
inflections in the latency ranges $90-160$ and 170 $230 \mathrm{msec}$, respectively, poststimulus. Mean latencies for N1 and P2 were 130 and $200 \mathrm{msec}$, respectively.

The peak-to-peak amplitude of the vertex potential (N1-P2) was extracted from the average EP for each condition and subjected to a repeated measures analysis of variance. The mean amplitudes for the three conditions were $7.2,4.4$, and $4.3 \mu \mathrm{V}$ for the $1,2 \mathrm{i}$, and $2 c$ conditions, respectively $[F(2,10)=6.54$, $\mathrm{p}<.01$ ]. An orthogonal comparisons analysis (Keppel, 1973) indicated the amplitude of N1-P2 to be significantly larger for the first stimulus of a pair irrespective of the laterality of the second [contrast coefficients $1-1 / 2-1 / 2: F(1,10)=13.20, p<.01]$, and this response decrement to be as equally developed for contralateral stimulation as it is for ipsilateral $(01-1: F<1)$. This orthogonal analysis was supported by a Newman-Keuls multiple comparisons analysis which showed significant refractoriness for both $2 \mathrm{i}$ and $2 \mathrm{c}$ in relation to $1(2, \mathrm{p}<.05$ in both cases) but no reliable difference between $2 \mathrm{i}$ and $2 \mathrm{c}$ $(p>.7)$. In passing, it is noted that, although stimulation details are not equivalent, the degree of refractoriness obtained here $(-4.4 \mathrm{~dB})$ is nonetheless comparable with the figure of $-4.8 \mathrm{~dB}$ that can be derived from the visual recovery function of Gjerdingen and Tomsic (1970) for a 1 -sec ISI in relation to a 10-sec ISI.

A problem with peak-to-peak measures of EP amplitude, such as N1-P2, is that component peaks may be varying independently. Accordingly, base-to-peak measures of both N1 and P2 amplitude were obtained and analyzed. Typically, in contemporary EP research, averaged prestimulus activity is used as the baseline in assessing base-to-peak amplitudes of individual EP components. However, because our initial concern was preoccupied with the peak-to-peak feature N1-P2, prestimulus activity had not been collected. In lieu of a prestimulus baseline, base-to-peak amplitude measures were made with respect to the following two baselines: (1) average vertex activity $0-25 \mathrm{msec}$ poststimulus (Walter, 1964, indicates a latency in excess of $25 \mathrm{msec}$ for the activation of the nonspecific cortex by a visual stimulus), and (2) amplifier $0 \mathrm{~V}$ baseline. Two two-factor repeated-measures ANOVAs were carried out with components (N1, P2) and treatments $(1,2 i, 2 c)$ as the factors. The results for both baselines indicated that $\mathrm{N} 1$ and P2 were behaving identically, with the interaction between treatments and components proving not significant, with $F$ ratios of $<1$ in both cases.

$\mathrm{N} 1$ and P2 latencies were also measured and analyzed. Repeated-measures ANOVAs revealed that both components were significantly later for the first stimulus of the pair [comparison $1-1 / 2-1 / 2: F(1,10)$ $=4.57, \mathrm{p}<.05$, for $\mathrm{N} 1$ and $\mathrm{F}(1,10)=14.57$, $p<.001$, for $P 2]$, but again laterality of the second stimulus appears to have little effect [comparison $01-1: \mathrm{F}<1$ for $\mathrm{N} 1$ and $\mathrm{F}(1,10)=2.33$, n.s. for P2].

\section{DISCUSSION}

The present data indicate that the visual vertex potential (N1-P2) is depressed following a recent prior stimulus irrespective of whether the second stimulus is delivered to the eye ipsilateral or contralateral to the first stimulus. In the logic of the dichoptic viewing paradigm, this strongly suggests that the source of this "fatigue" is central. As far as identifying which cell populations are involved, it is possible, for instance, that the refractoriness is a property of the cell populations responsible directly for the electrogenesis of N1-P2. However, any cortical cell population earlier in the information processing sequence could, of course, also be responsible.

Long (1979), in a recent critical review of the dichoptic viewing logic, argues that precortical mechanisms may not, after all, necessarily be precluded by a positive dichoptic result such as ours. He would argue that our result does not preclude the possibility that refractoriness is a precortical phenomenon whose consequences affect binocular levels. In other words, it may be that, in the present data, the refractoriness experienced by the contralateral second stimulus is the result of "fatigued" precortical cells on the ipsilateral side maintaining a sustained inhibition of higher binocular centers.

Strictly, then, it may well be that all one can conclude from the present data is that refractoriness transfers interocularly. The authors, however, have reservations over Long's caveat in the present context. We are, for instance, unaware of independent electrophysiological evidence for precortical corticopetal inhibition in the visual system operating over the present extended time-scale of the temporal recovery of the EP. A second difficulty for such a hypothetical precortical refractoriness is that, even assuming such a mechanism, it is hard to see that a stimulus which experiences both the refractory neural elements themselves and subsequently the inhibited binocular levels (i.e., the ipsilateral second stimulus, 2i) will not be more "disadvantaged" than a stimulus only experiencing the latter (i.e., the second stimulus contralateral to the refractory cells, $2 \mathrm{c}$ ). The present data, however, give no such suggestion, with N1-P2 amplitude for $2 \mathrm{i}$ and $2 \mathrm{c}$ being statistically indistinguishable. Although failure to reject the null hypothesis does not, of course, prove it, the low level of significance for the $2 \mathrm{i}$ vs. $2 \mathrm{c}$ comparison and the essentially identical estimates of N1-P2 amplitude for these two conditions (what little difference there is being in the "wrong" directon) do strongly suggest 
that the contralateral second stimulus has no particular advantage over the ipsilateral one.

Although latency effects have generally received less attention in studies of the temporal recovery of the EP, such effects have been frequently noted in "short-term habituation" paradigms (e.g., Fruhstorfer et al., 1970; Maclean, Ohman, \& Lader, 1975; Wastell \& Kleinman, Note 1), where a "forward latency shift" is generally found to accompany the amplitude decrement as the stimulus is repeated. The present data, in showing a similar latency facilitation, are thus compatible with this earlier work. The finding of a forward latency shift accompanying the amplitude depression is, however, a curious one from a simple refractoriness interpretation of these effects, which would tend to predict a latency delay rather than facilitation to be associated with the refractory state. These latency effects do become, however, more explicable from considerations of the temporal predictability of stimuli in the experiment. The forward latency shift can probably be attributed to a facilitation of information processing associated with the greater temporal predictability of $2 \mathrm{i}$ and $2 \mathrm{c}$ due to the close temporal proximity of 1 . Certainly reaction time data (Näätänen \& Merisalo, 1977) evidences such a speeding-up of processing in relation to the temporal predictability of stimuli.

\section{REFERENCE NOTE}

1. Wastell, D. G., \& Kleinman, D. Fast habituation of late componentry of the visual evoked potential in man. Manuscript submitted for publication, 1979.

\section{REFERENCES}

Bess, J. C., \& Ruнm, H. B. Recovery cycle of the acoustically evoked potential. Journal of Speech and Hearing Research, $1972,14,507-517$.
Davis, H., Mast, T., Yoshie, N., \& Zerlin, S. The slow response of the human cortex to auditory stimuli: Recovery process. Electroencephalography and Clinical Neurophysiology, $1966,21,105-113$.

Davis, H., Osterhammel, R. A., Weir, C. C., \& Gjerdingen, D. B. Slow vertex potentials: Interactions among auditory, tactile, electric and visual stimuli. Electroencephalography and Clinical Neurophysiology, 1972, 33, 537-545.

Fruhstorfer, H., Soveri, P., \& Jarvilehto, T. Short-term habituation of the auditory evoked response in man. Electroencephalography and Clinical Neurophysiology, 1970, 28, 153-161.

GJerdingen, D. B., \& Tomsic, R. Recovery functions of human cortical potentials evoked by tones, shocks, vibration and flashes. Psychonomic Science, 1970, 19, 228-229.

KEPPEL, G. Design and analysis: $A$ researcher's handbook. Englewood Cliffs, N.J: Prentice-Hall, 1973.

Long, G. M. The dichoptic viewing paradigm: Do the eyes have it? Psychological Bulletin, 1979, 86, 391-403.

Maclean, V., Ohman, A., \& Lader, M. Effects of attention, activation and stimulus regularity on short-term 'habituation' of the averaged evoked response. Biological Psychology, 1975, 3, 57.69.

Nätränen, R., \& Merisalo, A. Expectancy and preparation in simple reaction time. In S. Dornic (Ed.), Attention and performance VI. Hillsdale, N.J: Erlbaum, 1977.

Szengothai, J., \& Arbib, M. A. Conceptual models of neural organization. Cambridge, Mass: M.I.T. Press, 1975.

TURveY, M. T. On peripheral and central processes in vision: Inferences from an information processing analysis of masking with patterned stimuli. Psychological Review, 1973, 80, 1-52.

WALTER, W. G. The convergence and interaction of visual, auditory and tactile responses in the human non-specific cortex. Annals of the New York Academy of Sciences, 1964, 112, 320-361.

WASTELL, D. G. Attention and the habituation of human brain potentials. Unpublished doctoral thesis, Durham University, 1978. (a)

Wastell, D. G. The application of low-pass linear filters to evoked potential data: Filtering without phase distortion. Electroencephalography and Clinical Neurophysiology, 1978, 46, 355-356. (b)

(Received for publication October 3, 1979; revision accepted November 29,1979 .) 